\title{
Intraocular lens implantation by surgeons in training
}

\author{
G. A. SUTTON \\ From the Birmingham and Midland Eye Hospital, Birmingham
}

SUMMARY This paper is a retrospective study of 101 consecutive intraocular lens implantations with the Binkhorst 4 and 2-loop type lenses. The surgery represents a limited series of implant operations carried out by 10 surgeons of senior registrar grade on a programme of higher surgical training. When pre-existing adverse factors were excluded, the percentage of best visual results achieved was less than the best acuity levels reported by more experienced surgeons. The incidence of complications which could be related to surgical factors was found to be higher.

The 101 cases reported here are the results of intraocular lens implant operations carried out by 10 ophthalmic surgeons in training. The purpose of the study was to examine the visual results, identify the complications encountered, and to establish if possible the main factors which influenced the final visual outcome.

\section{Materials and methods}

With the exception of a small number of extracapsular lens extractions, which are discussed below, the majority of cataractous lenses were removed by a standard intracapsular cryoextraction technique. Ninety-six Binkhorst 4-loop lenses and 5 Binkhorst 2-loop lenses were implanted. Two surgeons carried out $58 \%$ of all the implant operations, and 2 other surgeons accounted for an additional $23 \%$ of the total. The remaining 6 surgeons carried out 20 cases in the total study. Surgery was carried out under general anaesthesia in all cases with the use of routine microsurgical techniques. The time of follow-up covered a period from 12 months up to a maximum of 6 years, and all except 4 eyes were available for follow-up evaluation. The corrected visual acuities were recorded in each case.

\section{Results}

After intraocular lens implantation $63 \%$ attained a visual acuity of $6 / 9$ or better. An additional $9 \%$ attained an acuity of $6 / 12$. The sum of these good visual results was $72 \%$ of the total. Three of these eyes had planned extracapsular lens extractions with

Correspondence to $\mathrm{Mr}$ G. A. Sutton, Birmingham and Midland Eye Hospital, Church Street, Birmingham B3 2NS. a Binkhorst 2-loop lens insertion, while the remainder all had intracapsular lens extractions and Binkhorst 4-loop lens implants.

In $25 \%$ of the total number of cases the visual outcome was considered poor, with a spectrum of visual results ranging from $6 / 18$ to hand movements, and in 1 case there was finally no perception of light.

\section{Discussion}

When those cases which had a poor visual outcome for reasons unrelated to the surgical procedure are excluded, a good visual result following implant surgery was achieved in $79 \%$. This compares with a $94 \%$ good visual result recorded by Roper-Hall ${ }^{1}$ with a follow-up period of more than 2 years. $\mathrm{Kratz}^{2}$ reports a $94 \%$ good visual outcome, but his follow-up time is significantly shorter. Dallas ${ }^{3}$ found that $67 \%$ of eyes after plain cataract extraction achieved a vision of $6 / 12$ or better.

Though positive pressure ventilation was the preferred form of general anaesthesia, there was no evidence from examining this series of patients that spontaneous ventilation adversely influenced the visual outcome. A bulging forward of the iris plane or vitreous loss was uncommon with either form of anaesthesia, and operative hyphaemas or subsequent poor wound security could not be attributed to the prevailing form of anaesthesia.

No correlation was found between the visual results obtained and the pre-existence of poor general health, including chronic bronchitis, congestive cardiac failure, or maturity onset diabetes; patients with a diastolic blood pressure of 100 $\mathrm{mmHg}$ or over had an increased incidence of operative hyphaemas. Striate keratopathy persisting 
for 3 or more days after surgery was considered probably due to operative trauma. The main factor in this respect was thought to be endothelial touch by the implant or associated instrumentation at the time of implantation. A corneal section was used in 53 eyes, while a limbal incision beneath a conjunctival flap was used in 48 eyes; striate keratopathy was found in $47 \%$ of the corneal section eyes while it occurred in $51 \%$ of the limbal sections. Among the good visual results corneal and limbal sections were used in $57 \%$ and $43 \%$ respectively.

\section{COMPLICATIONS}

In $9 \%$ of the total number of cases complications were encountered which were considered not peculiar to intraocular lens implantation. These included pre-existing ocular pathology and some postoperative complications described below. One patient had pre-existing optic atrophy due to earlier radiation for an orbital neoplasm. An extracapsular lens aspiration in 1 case following childhood trauma, and an additional case with a congenital uniocular cataract, predictably resulted in 2 amblyopic eyes with consequently poor vision. One patient was found to have senile macular degeneration. In the postoperative period 2 eyes developed retinal detachments with poor long-term visual acuities. In 1 eye a central retinal vein occlusion led to poor vision. Another patient banged her eye 1 week after successful implant surgery and ruptured the globe with gross loss of intraocular contents. An additional eye was complicated by epithelialisation of the anterior chamber, and this eye was enucleated.

In a further $6 \%$ complications which could possibly be related to intraocular lens implantation were found. Four eyes with protracted uveitis developed secondary membrane formation. At surgery in three of these eyes an anterior vitrectomy following vitreous loss was necessary. On reviewing these eyes with uveitis there was a delay in instituting adequate topical treatment in the majority of cases.

Cystoid maculopathy was found by clinical examination in $2 \%$ to account for poor vision, which is less than the $12 \%{ }^{4}$ and $7 \%{ }^{3}$ reported, but equal to the incidence following plain intracapsular cataract extractions. ${ }^{5}$ It is possible, however, that cystoid maculopathy could have been present in some other cases concurrently with corneal oedema or membrane development and so evaded detection.

In the remaining $10 \%$ of cases endothelial decompensation occurred, and this complication was attributed predominantly to factors related to intraocular lens implantation. Half of this group initially enjoyed good vision, but after a period ranging from 11 months to 44 months their vision deteriorated from an acuity of 6/9-6/12 to 6/24 hand movements, due to corneal endothelial decompensation.

Two eyes with endothelial decompensation were additionally complicated by concurrent uveitis with membrane development and secondary glaucoma. Another eye with endothelial decompensation also developed glaucoma. In 3 of these eyes the lens implant was surgically removed. This course of action would seldom be advocated now.

In this series, the level of best visual acuity attained is lower than that of experienced surgeons. The incidence of complications will predictably be higher in the first 20 cases than in the next series. ${ }^{6} \mathrm{~A}$ poor visual outcome was found when an intraocular lens implantation followed a lens extraction complicated by vitreous loss. It should be noted that in half of the eyes in which endothelial decompensation was the cause of poor vision the onset of endothelial dysfunction was delayed for between 11 and 44 months after the original surgery. With a continually improving technique it is hoped that the incidence of endothelial decompensation will be greatly reduced.

I thank Mr W. Martin-Walker for encouraging me to review these patients.

\section{References}

${ }^{1}$ Roper-Hall MJ. Trans Ophthalmol Soc UK 1979; in press. ${ }^{2} \mathrm{Kratz}$ RP. The consecutive implantation of 250 shearing intraocular lenses. Contact and Intraocular Lens Journal 1979; 5: 123-9.

${ }^{3}$ Dallas NL. Comparison of iris clip lens results with plain intra-capsular extraction. Proc $R$ Soc Med 1976; 69: 903-5. ${ }^{4}$ Binkhorst CD, Leonard PAM. Results in 208 iris-clip pseudophakos implantations. Am J Ophthalmol 1967; 64: 947-56.

${ }^{5}$ Easty D, Dallas NL, O'Malley R. Aphakic macular oedema following prosthetic lens implantation. $\mathrm{Br} J$ Ophthalmol $1977 ; 61$ : 321-6.

${ }^{6}$ Byron HM. Intra-capsular implant surgery for the beginner. Contact Intraocular Lens Journal 1977; 3: 66-70. 